\title{
Zentrale Ethikkommission der SAMW: auf weitere 25 Jahre!
}

Geht es wohl den medizinisch-ethischen Richtlinien der Schweizerischen Akademie der Medizinischen Wissenschaften SAMW wie dem «Ulysses»: vielen bekannt, aber von wenigen wirklich gelesen? Eine Frage, die wir uns oft stellen.

Tatsache ist, dass die Richtlinien eine hohe Akzeptanz und Glaubwürdigkeit haben, den aktuellen Stand der ethischen Diskussion reflektieren und durch ein breites Vernehmlassungsverfahren bei der Ärzteschaft auch die Stimmen der Basis einbeziehen. Gerade in Gebieten, in denen Gesetze (noch) fehlen, stellen sie wichtige Leitplanken dar, die helfen, in ethisch und menschlich schwierigen Situationen gute Entscheide zu treffen.

Die mit der Erarbeitung der Richtlinien betraute Zentrale Ethikkommission (ZEK) hat seit ihrer Gründung vor 25 Jahren eine beeindruckende Arbeit geleistet, und zwar immer ehrenamtlich (einziger «Lohn» ist jeweils ein Nachtessen nach Abschluss der Arbeiten innerhalb einer Subkommission).

Anfragen zur Mitarbeit in der ZEK oder in einer ihrer Subkommissionen werden erfreulicherweise kaum je mit einer Absage beantwortet.
Es scheint, dass die thematische Arbeit, die mit den Richtlinien in einem sichtbaren und brauchbaren Resultat mündet, attraktiv und lohnend ist.

Die aktuelle Kritik von verschiedener Seite, dass es in der Schweiz momentan etwas gar viele Ethikkommissionen gebe und der Ethikbegriff teilweise inflationär verwendet werde, mag ein Stück weit berechtigt sein. Mit der fortschreitenden Technologisierung der Medizin stellen sich jedoch im ärztlichen und pflegerischen Alltag immer neue Fragen, bei denen eine ethische Auseinandersetzung unabdingbar ist. Der ZEK werden auch in den kommenden Jahren die Themen nicht ausgehen!

Margrit Leuthold, Generalsekretärin der SAMW

PS. Aus Anlass des 25-Jahre-Jubiläums wirft der Präsident der ZEK, Prof. Michel Vallotton, einen Blick in die Vergangenheit und in die Zukunft (S. 1939-42).

Feiern Sie mit uns am 23. September 2004 das 25jährige Bestehen der ZEK im Rahmen eines halbtägigen Symposiums (Programm auf S. 1943), welches dem Thema «Neuroethik» gewidmet ist. Und lesen Sie den Ulysses. Beides lohnt sich! 


\section{Commission Centrale d'Ethique de I'Académie Suisse des Sciences Médicales: vers un prochain quart de siècle!}

En est-il des Directives médico-éthiques de l'Académie Suisse des Sciences Médicales (ASSM) comme des récits de l'Ulysse de Joyce: bien connus, mais en vérité peu lus? La question se pose parfois.

Cela dit, les directives sont bien acceptées et jouissent d'une grande crédibilité. Elles reflètent l'état actuel du débat sur l'éthique tout en intégrant, par une large procédure de consultation du corps médical, les voix de la base.

Tout spécialement dans les domaines où il manque (encore) des lois, ces directives sont d'importants paradigmes permettant de soutenir les bonnes décisions à prendre dans des situations éthiquement et humainement difficiles.

Depuis sa création il y a 25 ans, la Commission Centrale d'Ethique (CCE) a fourni un travail remarquable dans l'élaboration de ces directives, toujours à titre bénévole (le seul «salaire» consistant en un repas pris en commun après les travaux de sous-commissions).

Une proposition de collaboration dans la CCE ou dans l'une de ses sous-commissions se heurte rarement à un refus. Il est certes enrichissant et agréable de travailler thématiquement sur des directives qui débouchent sur un résultat tangible et profitable.

Les critiques que l'on entend parfois selon lesquelles la Suisse compte actuellement un trop grand nombre de commissions d'éthique et que ce terme d'éthique commence à être galvaudé peuvent dans une certaine mesure se comprendre. Mais les progrès techniques de la médecine posent dans le quotidien des soins des questions toujours nouvelles pour lesquelles une réflexion éthique est indispensable. Ce ne sont pas les débats de ce type qui devraient manquer à la CCE ces prochaines années!

Margrit Leuthold, secrétaire générale de l'ASSM

P.-S. A l'occasion de cet anniversaire, le Prof. Michel Vallotton, président de la CCE, jettera un regard sur le passé (cf. pages 1935-8).

Célébrez avec nous, le 23 septembre prochain, les 25 ans de la CCE lors d'un symposium d'une demi-journée qui sera consacré à la «neuroéthique» (cf. programme sur la page 1943), sans oublier de lire les aventures d'Ulysse. Les deux ne sont pas incompatibles, bien au contraire! 